\title{
BMJ Open Efficacy of perioperative immunonutrition in visceral surgery: an umbrella review protocol
}

\author{
Karem Slim (D , ${ }^{1}$ Flora Badon, ${ }^{1}$ Charles-Hervé Vacheron, ${ }^{2,3}$ Chadli Dziri, ${ }^{4}$ \\ Thomas Marquillier (i) ${ }^{5,6}$
}

To cite: Slim K, Badon F, Vacheron $\mathrm{C}-\mathrm{H}$, et al. Efficacy of perioperative immunonutrition in visceral surgery: an umbrella review protocol. BMJ Open 2021;11:e053851. doi:10.1136/ bmjopen-2021-053851

- Prepublication history for this paper is available online To view these files, please visit the journal online (http://dx.dol org/10.1136/bmjopen-2021053851).

Received 29 May 2021 Accepted 01 September 2021

D) Check for updates

(c) Author(s) (or their employer(s)) 2021. Re-use permitted under CC BY-NC. No commercial re-use. See rights and permissions. Published by BMJ.

${ }^{1}$ Department of Digestive Surgery, CHU, Clermont-Ferrand, France

${ }^{2}$ Department of Anaesthesiology, Hospices Civils de Lyon, Lyon, France

${ }^{3}$ Department of Biostatistics, Hospices Civils de Lyon, Lyon, France

${ }^{4}$ Director of Honoris Medical Simulation Center, Tunis, Tunisia

${ }^{5}$ School of Dentistry, Department of Pediatric Dentistry, University of Lille, Lille, France

${ }^{6} \mathrm{Health}$ Education and Practices Laboratory (LEPS UR 3412),

Sorbonne North Paris University - Bobigny Campus, Bobigny, France

Correspondence to

Dr Karem Slim;

kslim@chu-clermontferrand.fr

\section{ABSTRACT}

Introduction Immunonutrition (IN) is generally used before major visceral surgery with the intent to reduce postoperative complications, especially infectious ones. However, the conclusions of published meta-analyses are conflicting. The purpose of this review is to synthesise the data of published systematic reviews on the effectiveness of IN.

Methods and analysis This protocol follows the Preferred Reporting Items for Systematic Reviews and Metaanalyses Protocols guidelines. This is an umbrella review of systematic reviews comparing IN (delivered orally 5-7 days preoperatively) with normal diet or isocaloric isonitrogenous feeding before visceral surgery performed on any of several viscera (colorectum, stomach, pancreas, liver, oesophagus). We search the systematic reviews included in the main bibliographic databases. To assess the efficacy of IN, several outcomes will be considered: the main outcome is infectious complications (surgical site infections, pulmonary infections or urinary infections) and secondary outcomes are overall morbidity, hospital length of stay and mortality. Identified reviews will be screened by two independent assessors. The methodological quality of relevant included reviews will be assessed using A MeaSurement Tool to Assess systematic Reviews (AMSTAR) instrument. The data extracted from included reviews will be synthesised using the r-Metafor package considering separate groups according to the viscus of interest. Publication bias will be evaluated, and subgroup analyses will be performed according to the quality of studies and preoperative nutritional status.

Ethics and dissemination An umbrella review based on published data from systematic reviews needs no ethical approval. Furthermore, no patient will be involved in the review. Once terminated, the review will be submitted for publication in an open access journal to ensure wide dissemination of the findings.

PROSPERO registration number CRD42021255177.

\section{INTRODUCTION}

Major digestive surgery is associated with significant postoperative morbidity, including infectious complications. To improve the postoperative course of surgery patients, some advocate the use of preoperative immunomodulating nutrition. Immunomodulating nutrition or immunonutrition (IN) is

\section{Strengths and limitations of this study}

- The strengths of this umbrella review are its comprehensiveness.

- The methodology followed the Preferred Reporting Items for Systematic Reviews and Meta-analyses 2020 guidelines.

- The limitations are related to the quality of included systematic reviews.

- Heterogeneity of included systematic review is a possible limitation which will be addressed.

a nutritional support using glutamine (Gln), arginine (Arg), omega-3 polyunsaturated fatty acids (PUFAs) and nucleotides. IN acts by modulating the inflammatory response and counteracts postoperative immune impairment, mainly in cases of major surgery. After the early studies by Braga et al, ${ }^{1}$ several subsequent studies suggested that IN could reduce the rate of complications, particularly postoperative infectious complications. This led the European Society of Parenteral and Enteral Nutrition (ESPEN) ${ }^{2}$ and French (Société Française de Nutrition Entérale et Parentérale - SFNEP) ${ }^{3}$ societies for nutrition to recommend the administration of immunonutrition (IN) before surgery in malnourished patients. However, despite the large amount of literature data, the benefit of preoperative IN in major digestive surgery or in malnourished patients remains controversial, especially when surgery is performed in the setting of an enhanced recovery programme, ${ }^{45}$ now considered as the gold standard of surgical care. ${ }^{6}$

Besides the enhanced recovery setting, the most recent meta-analysis of 16 trials (1387 patients with gastrointestinal cancers) concluded that IN halved the number of infectious complications compared with controls (isocaloric isonitrogenous feed or normal diet). ${ }^{7}$ Another meta-analysis published in 
the same year yielded findings that should be taken with caution given the high heterogeneity. ${ }^{8}$

To the best of our knowledge, there is no umbrella review of published meta-analyses on this topic.

The aim of this umbrella review is to evaluate the effect of preoperative IN treatment on postoperative morbidity in elective visceral surgery, by synthesising the data of published systematic reviews on the effectiveness of IN compared with normal diet. To this end, the review will address the following three questions:

1. Does IN reduce overall postoperative morbidity especially postoperative infectious complications?

2. In what surgical specialties could IN play a role?

3. In what circumstances (nutritional status, disease, type of care, eg, enhanced recovery programmes), could IN be useful?

\section{METHODS}

\section{Protocol design}

This protocol follows the Preferred Reporting Items for Systematic Reviews and Meta-analyses (PRISMA) Protocols guidelines. ${ }^{9}$ The review will follow the Joanna Briggs Institute methodology for umbrella reviews ${ }^{10}$ and the updated PRISMA. ${ }^{11}$ The review will start on 1 June 2021, and will end on 1 October 2021.

\section{Inclusion criteria}

\section{Participants}

This review will include systematic reviews (with or without meta-analysis) evaluating IN in major digestive surgery, that is, liver, oesophageal, gastric, pancreatic or colorectal surgeries. A systematic review will be defined as a paper with a PICO statement (population, intervention, comparator, outcome), a search strategy, inclusion criteria or inclusion of randomised or non-randomised comparative studies (such as case-control studies). Reviews of qualitative studies or narrative reviews will be excluded.

\section{Intervention}

This review will consider only preoperative IN delivered orally $5-7$ days before surgery

\section{Comparators}

IN with nutrients such as Gln, Arg, omega-3 PUFAs and nucleotides versus normal diet or isocaloric isonitrogenous feeding.

\section{Outcomes}

This review will consider the following postoperative outcomes: the primary outcome is infectious complications (surgical site infections, pulmonary infections and urinary infections), secondary outcomes are overall postoperative complications, hospital length of stay and postoperative mortality.

\section{Search strategy}

The following databases will be searched: Medline, PICO, Cochrane database for systematic reviews, Scopus,
PROSPERO International prospective register of systematic reviews and Google. The references listed in each retrieved article will be manually searched. The MeSH terms used will be: (immunonutrition) AND (surgery) with the filters (systematic reviews) OR (meta-analysis). The search strategy will also be adapted to each database. Generally, the keyword 'immunonutrition' will be used in the other databases. If necessary, the corresponding authors of the included systematic reviews will be contacted to inquire about missing data regarding the main endpoints.

Only systematic reviews published in English, French, German, Italian or Spanish will be selected. We will contact the authors of protocols included in PROSPERO that are terminated but not yet published, to ask them about their data and results.

\section{Review selection}

The titles and abstracts of all identified reviews will be screened by two independent assessors against the inclusion and exclusion criteria. Any disagreement will be resolved by consensus or with a third assessor. The full texts of relevant reviews will then be assessed independently by two independent assessors against the inclusion criteria. The reasons for excluding a review are summarised in the flow chart. The selection process is presented in a PRISMA flow chart (figure 1). The included reviews will be analysed to describe the primary studies overlap within the reviews and the corrected covered area index will be calculated. ${ }^{12}$ A corrected covered area index of $0-5$ indicates a slight overlap, $6-10$ a moderate overlap, 11-15 a high overlap and $>15$ a very high overlap.

We will extract a dataset from each included review: first author, year of publication, country, objective of the review, inclusion/exclusion criteria, search strategy and results, number of patients and included studies, type of studies, methodological quality assessment, interventions (IN nutrients, preoperative, perioperative), comparators, outcomes, results and conclusions.

\section{Assessment of methodological quality}

Methodological quality of all included reviews will be assessed by two independent assessors using A MeaSurement Tool to Assess systematic Reviews (AMSTAR) ${ }^{13}$ in the case of systematic review of randomised trials, and alternatively AMSTAR-2 ${ }^{14}$ in the case of systematic review of randomised and non-randomised trials. The review quality will be presented in a table summarising the review characteristics. The level of evidence related to each included review will also be presented according to the Grading of Recommendations, Assessment, Development and Evaluations (GRADE) ${ }^{15}$ This approach will help clarify the influence of the methodological quality and the level of evidence of the included reviews on the comprehensiveness and validity of this umbrella review. 


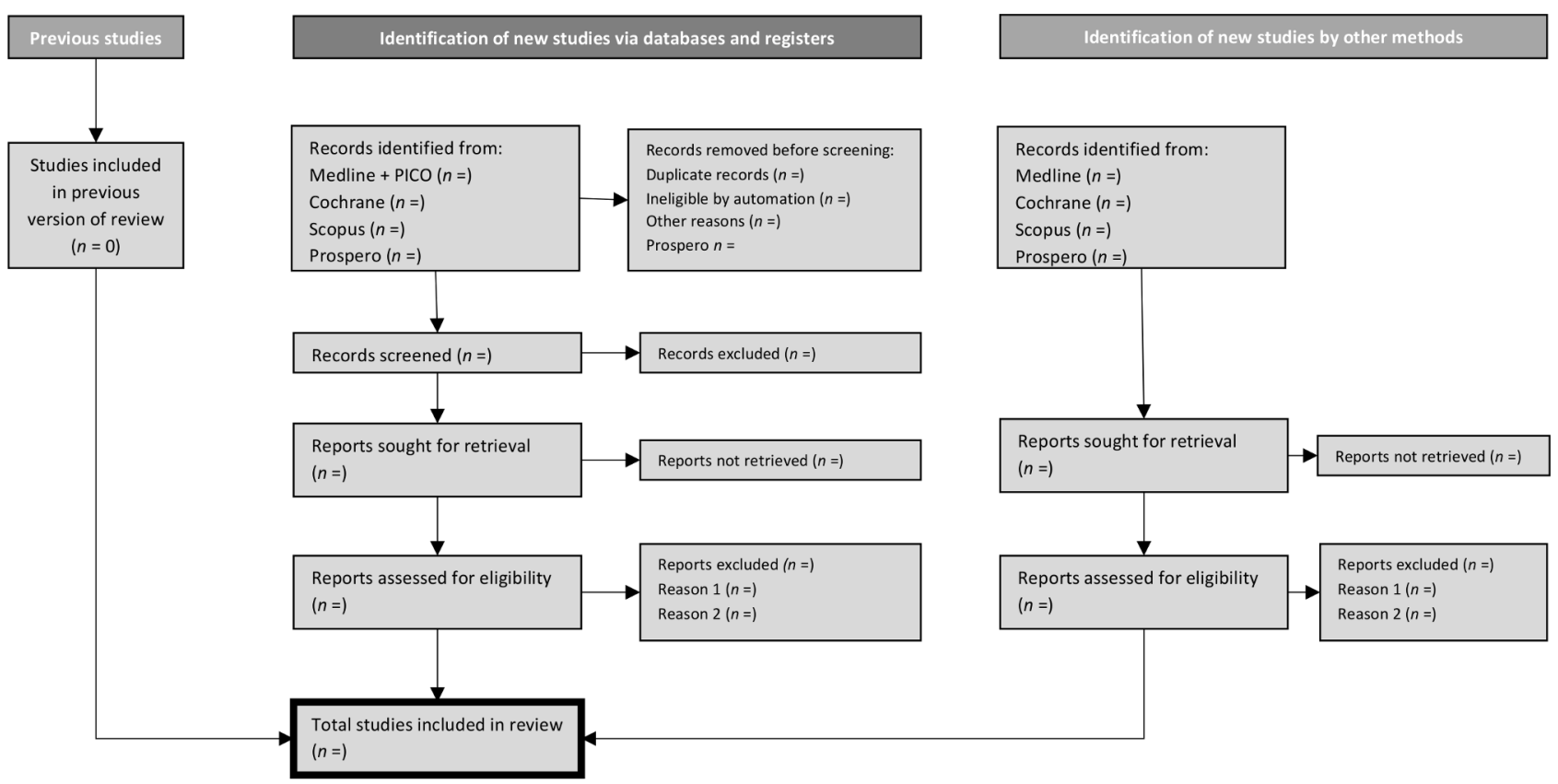

Figure 1 PRISMA 2020 flow chart. PICO, population, intervention, comparator, outcome; PRISMA, Preferred Reporting Items for Systematic Reviews and Meta-analyses.

\section{Data synthesis and statistical analysis}

We will separately consider five groups according to the surgical specialty: liver, oesophageal, gastric, pancreatic or colorectal surgery. The data of each group will be synthesised with respect to the outcomes; with subsequent analyses, whether the patients were malnourished or not. We will screen the included review against the definition of malnutrition. The types of immunonutrients will also be considered for a sensitivity analysis.

If available after random models, the pooled effect sizes of included reviews will be extracted. If not, the original data will be extracted, and the effect size calculated with its $95 \%$ CI using a random model.

The r-Metafor package software will be used for this umbrella review. The random effects model will be used to estimate the OR or HR and 95\% CI. Otherwise, the Hedges adjusted g (corrected effect size) will be calculated. The $Q$ and $\mathrm{I}^{2}$ statistics will be calculated to test the heterogeneity. The degree of heterogeneity will be defined as considerable $\left(\mathrm{I}^{2}>75 \%\right)$ or substantial $\left(\mathrm{I}^{2}>50 \%\right)$. Publication bias will be evaluated using the Egger test and funnel plots. In the case of absence of quantitative data in the included reviews, only the direction of the effect and statistical significance will be provided.

Certainty of the evidence will be assessed using the GRADE approach, ${ }^{15}$ by calculating the absolute risk for treatment and control groups, estimated relative risk, and ranking of the quality of evidence based on the risk of bias, overlapping, heterogeneity, precision and risk of publication bias.

\section{FUNDING}

This umbrella review will be funded by the University Hospital of Clermont-Ferrand France and the Francophone Group for Enhanced Recovery After Surgery.

\section{ETHICS AND DISSEMINATION}

There is no ethical nor safety considerations with this umbrella review since it will use published data in metaanalyses. Publication is planned in an international Journal.

\section{PATIENT AND PUBLIC INVOLVEMENT}

No patient involved

\section{DISCUSSION}

To our knowledge, this will be the first umbrella review to assess the efficacy of IN before visceral surgery. The results of this review will help clinicians prescribe IN appropriately according to the surgical specialty. The role of IN in enhanced recovery programmes will be more firmly established. ${ }^{16}$

Contributors Guarantor of the review: KS. Conception, design (selection criteria), writing and corrections: KS, C-HV and TM. Search strategy, data extraction and analysis: $\mathrm{KS}$, $\mathrm{FB}, \mathrm{CH}-\mathrm{V}$ and $\mathrm{CD}$. Statistical expertise: $\mathrm{CH}-\mathrm{V}, \mathrm{CD}$. All authors approved the final version

Funding This study was funded by The University Hospital CHU Clermont-Ferrand. There is no award/grant number.

Competing interests KS declares interests in Sanofi, Merck, B-Braun and Coloplast.

Patient and public involvement Patients and/or the public were not involved in the design, or conduct, or reporting, or dissemination plans of this research.

Patient consent for publication Not required. 
Provenance and peer review Not commissioned; externally peer reviewed.

Open access This is an open access article distributed in accordance with the Creative Commons Attribution Non Commercial (CC BY-NC 4.0) license, which permits others to distribute, remix, adapt, build upon this work non-commercially, and license their derivative works on different terms, provided the original work is properly cited, appropriate credit is given, any changes made indicated, and the use is non-commercial. See: http://creativecommons.org/licenses/by-nc/4.0/.

\section{ORCID iDs}

Karem Slim http://orcid.org/0000-0002-1527-4691

Thomas Marquillier http://orcid.org/0000-0003-0188-3872

\section{REFERENCES}

1 Braga M, Vignali A, Gianotti L, et al. Immune and nutritional effects of early enteral nutrition after major abdominal operations. Eur J Surg 1996;162:105-12.

2 Weimann A, Braga M, Carli F, et al. ESPEN guideline: clinical nutrition in surgery. Clin Nutr 2017;36:623-50.

3 Chambrier C, Sztark F, Société Francophone de nutrition clinique et métabolisme (SFNEP). [French clinical guidelines on perioperative nutrition. update of the 1994 consensus conference on perioperative artificial nutrition for elective surgery in adults"]. $J$ Visc Surg 2012;149:e325-36.

4 Gupta R, Senagore A. Immunonutrition within enhanced recovery after surgery (ERAS): an unresolved matter. Perioper Med 2017;6:24

5 Thornblade LW, Varghese TK, Shi X, et al. Preoperative immunonutrition and elective colorectal resection outcomes. Dis Colon Rectum 2017;60:68-75.

6 Ljungqvist $\mathrm{O}$, de Boer HD, Balfour A, et al. Opportunities and challenges for the next phase of enhanced recovery after surgery. JAMA Surg 2021;156:775
7 Adiamah A, Skořepa $\mathrm{P}$, Weimann A, et al. The impact of preoperative immune modulating nutrition on outcomes in patients undergoing surgery for gastrointestinal cancer: a systematic review and metaanalysis. Ann Surg 2019;270:247-56.

8 Zhang B, Najarali Z, Ruo L, et al. Effect of perioperative nutritional supplementation on postoperative Complications-Systematic review and meta-analysis. J Gastrointest Surg 2019;23:1682-93.

9 Shamseer L, Moher D, Clarke M, et al. Preferred reporting items for systematic review and meta-analysis protocols (PRISMA-P) 2015: elaboration and explanation. BMJ 2015;350:g7647.

10 Aromataris E, Fernandez R, Godfrey CM, et al. Summarizing systematic reviews: methodological development, conduct and reporting of an umbrella review approach. Int $J$ Evid Based Healthc 2015;13:132-40.

11 Page MJ, McKenzie JE, Bossuyt PM, et al. The PRISMA 2020 statement: an updated guideline for reporting systematic reviews. BMJ 2021;372:n71.

12 Pieper D, Antoine S-L, Mathes T, et al. Systematic review finds overlapping reviews were not mentioned in every other overview. $J$ Clin Epidemiol 2014;67:368-75.

13 Shea BJ, Grimshaw JM, Wells GA, et al. Development of AMSTAR: a measurement tool to assess the methodological quality of systematic reviews. BMC Med Res Methodol 2007;7:10.

14 Shea BJ, Reeves BC, Wells G, et al. AMSTAR 2: a critical appraisal tool for systematic reviews that include randomised or nonrandomised studies of healthcare interventions, or both. BM 2017;358:j4008.

15 Guyatt GH, Oxman AD, Vist GE, et al. GRADE: an emerging consensus on rating quality of evidence and strength of recommendations. BMJ 2008;336:924-6.

16 Ljungqvist $\mathrm{O}$, de Boer HD, Balfour $\mathrm{A}$, et al. Opportunities and challenges for the next phase of enhanced recovery after surgery: a review. JAMA Surg 2021;156:775 